\title{
DEVELOPMENT AND IMPLEMENTATION OF PRODUCTION AREA OF AGRICULTURAL PRODUCT DATA COLLECTION SYSTEM BASED ON EMBEDDED SYSTEM
}

\author{
Lei $\mathrm{Xi}^{1}$, Wei Guo ${ }^{1}$, Yinchao Che ${ }^{1}$, Hao Zhang ${ }^{1}$, Qiang Wang ${ }^{1}$, Xinming \\ $\mathrm{Ma}^{1,2, *}$ \\ 1 College of Information and Management Science, Henan Agricultural University, \\ Zhengzhou, He Nan, China, 450002 \\ ${ }^{2}$ College of Agriculture, Henan Agricultural University, Zhengzhou, He Nan, China, 450002 \\ * Corresponding author, Address: College of Information and Management Science ,Henan \\ Agricultural University, 63 Agricultural Road, Zhengzhou, 450002, P. R. China, Tel:+86- \\ 371-63558388, Fax:+86-371-63558090,Email: xinmingma@126.com
}

Abstract: $\quad$ To solve problems in detecting the origin of agricultural products, this paper brings about an embedded data-based terminal, applies middleware thinking, and provides reusable long-range two-way data exchange module between business equipment and data acquisition systems. The system is constructed by data collection node and data center nodes. Data collection nodes taking embedded data terminal NetBoxII as the core, consisting of data acquisition interface layer, controlling information layer and data exchange layer, completing the data reading of different front-end acquisition equipments, and packing the data TCP to realize the data exchange between data center nodes according to the physical link (GPRS / CDMA / Ethernet). Data center node consists of the data exchange layer, the data persistence layer, and the business interface layer, which make the data collecting durable, and provide standardized data for business systems based on mapping relationship of collected data and business data. Relying on public communications networks, application of the system could establish the road of flow of information between the scene of origin certification and management center, and could realize the real-time collection, storage and processing between data of origin certification scene and databases of certification organization, and could achieve needs of long-range detection of agricultural origin.

Keywords: embedded system, data collection, middleware, Web Services

Please use the following format when citing this chapter:

Xi, L., Guo, W., Che, Y., Zhang, H., Wang, Q. and Ma, X., 2009, in IFIP International Federation for Information Processing, Volume 295, Computer and Computing Technologies in Agriculture II, Volume 3, eds. D. Li, Z. Chunjiang, (Boston: Springer), pp. 1769-1781. 


\section{INTRODUCTION}

The collection of agricultural production environment information is an important link to guarantee the quality and safety of agricultural products, and these information on the safety of agricultural products are also main source of data and parameters of pre-warning system and decision support systems(Meng, 2006). Some domestic and abroad research institutions have developed convenient terminal equipment of field environmental data collection and the corresponding software based on embedded systems(Joann, 1998), such as the United States StarPal company's HGIS system, which based on the PocketPC, could collect vector and attribute information of GPS location and soil sampling. China's Xiao Chunhua(Xiao, et al. 2007) has developed Embedded Wireless Remote Environmental Monitoring System based on the ARM7, which apply to multi-source analog signal acquisition. Zhou Guoxiang (Zhou, et al. 2005), a specific remote monitoring controller (ReMoC) based on GSM was developed for this situation to communicate field equipment or apparatus with the monitoring computer on a farm. Meng Zhijun (Meng, et al. 2005), based on DGPS, using embedded GIS development components, realized the integration of GPS and GIS functions under Pocket PC. Currently embedded data collection system emphasis more on integration capabilities: multi-source data collection, data transmission based on variety of physical link and providing data support for business systems (Kevin, et al. 2002; Meng, et al. 2003; Wang, et al. 2006; FAN, et al. 2007; Qiu, et al. 2005).

The research is for environmental monitoring origin of agricultural products. It uses embedded data terminals to construct data collection node, to complete the data collection of multi-sensor analog signals and multichannel serial signals. Based on GPRS / CDMA / Ethernet physical links, it realizes rapid data transmission. The data are preserved in the data center node. The data center node uses Web Services technology to provide data service interface, and to achieve loose coupling with business systems.

\section{SYSTEM DESIGN}

The system uses C / S model, which is constructed of data collection node and a data center node, the system physical structure show in Figure 1. Data collection nodes take embedded data terminal NetBoxII (NetBoX-II,2003) as the core, be constructed of data acquisition interface layer, controlling information layer and data exchange layer, complete the data reading of different front-end acquisition equipment, pack the TCP data package, and achieve data exchange between the data center nodes based on Physical link 
(GPRS / CDMA / Ethernet). Data center node is constructed of the data exchange layer, the data persistence layer, and the business interface layer, which achieves durable storage of collection data, and provides standardized data through the business interface layer for business systems.

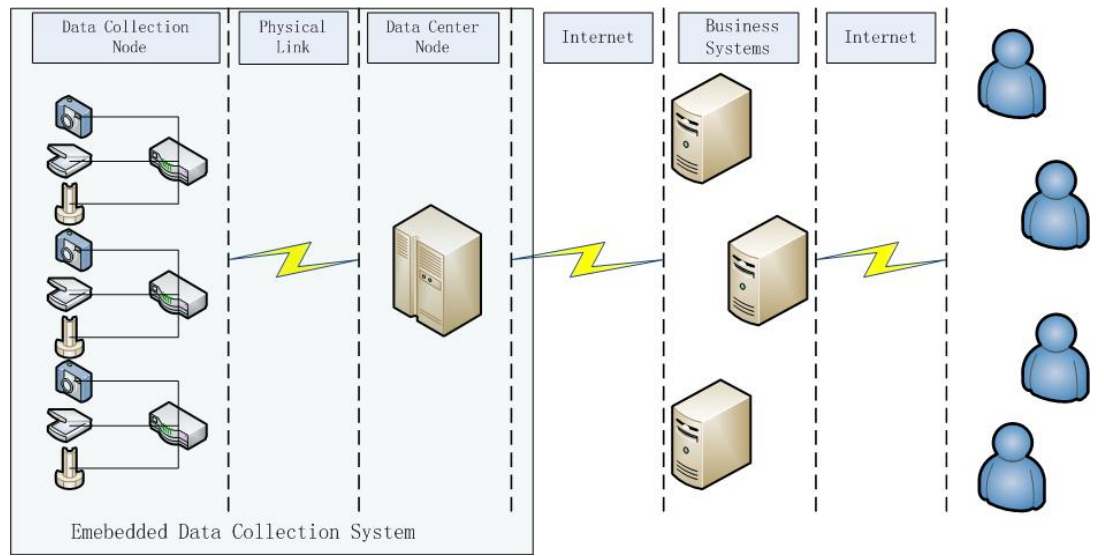

Figure 1. the system physical structure

\section{DATA CENTER NODE DESIGN}

\subsection{Module structure and implementation technology}

The main modules function as follows: (1) application modules, the system's main modules, make system run into the cycling of information, (2) configuration modules, complete configuration of network parameters, database parameters, security parameters, and the auto-launch parameters, (3) service launching Module, launch services, monitor the connection request of data collection nodes and complete the initialization of database interface, network interface and system buffering zone (4) database interface module, in charge of connection and lasting of system database; (5) network interface module, is responsible for monitoring network, establishing / releasing connect, send / receive TCP messages, (6) Costumer interactive module, responsible for receiving data acquisition request of data collection node, packaging / sending messages for data collection nodes, receiving / analyzing information from data collecting nodes and retrieving system database; (7) data service module, responsible for providing data accessing interface for the operational system. Data center node module structure shown in Figure 2. 


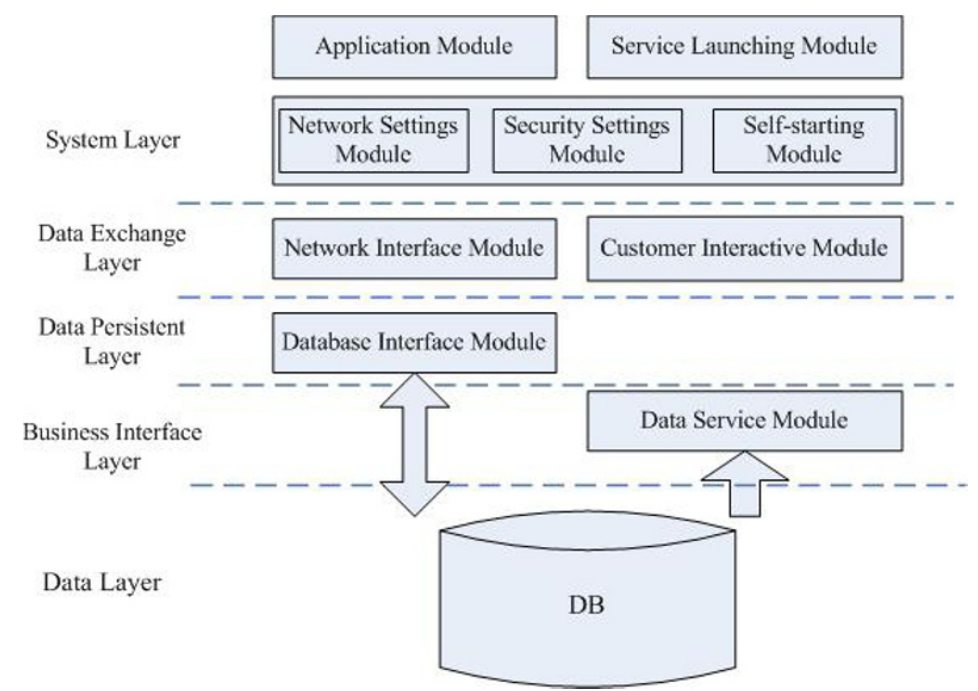

Figure 2. Data center node module structure

Data center node using windows 2003 server, oracle $10 \mathrm{~g}$ as platform for data, VC.NET 2003 developing the data exchange layer, data lasting layer and system layer. J2EE technology is used to achieve business interface layer. The application services and web services containers adopt BEA Weblogic 9.2.

\subsection{Data Model}

Core data is constructed of metadata and special topic data, the relationship model of the main data tables show in Figure 3. Special topic data are actually measured data of various types of objects, such as the latitude and longitude data of sampling points and soil nutrient data. Metadata are used to describe the relationship between data, including (1) Data Acquisition Category Table, describing the type of the system data acquisition. Data collection domain is the way of describing the initiation of data collection from the point of the data center node: the active approach, launched by the central node of data, data collection nodes to receive data acquisition orders; passive approach, initiated by the joint data collection, data center node passively receive data acquisition request, (2) table of thematic data categories, is the description of thematic data, and the entity table specifying the data storage. (3) Table of data acquisition categories and thematic data relationship, describing the relevance of the thematic data. 


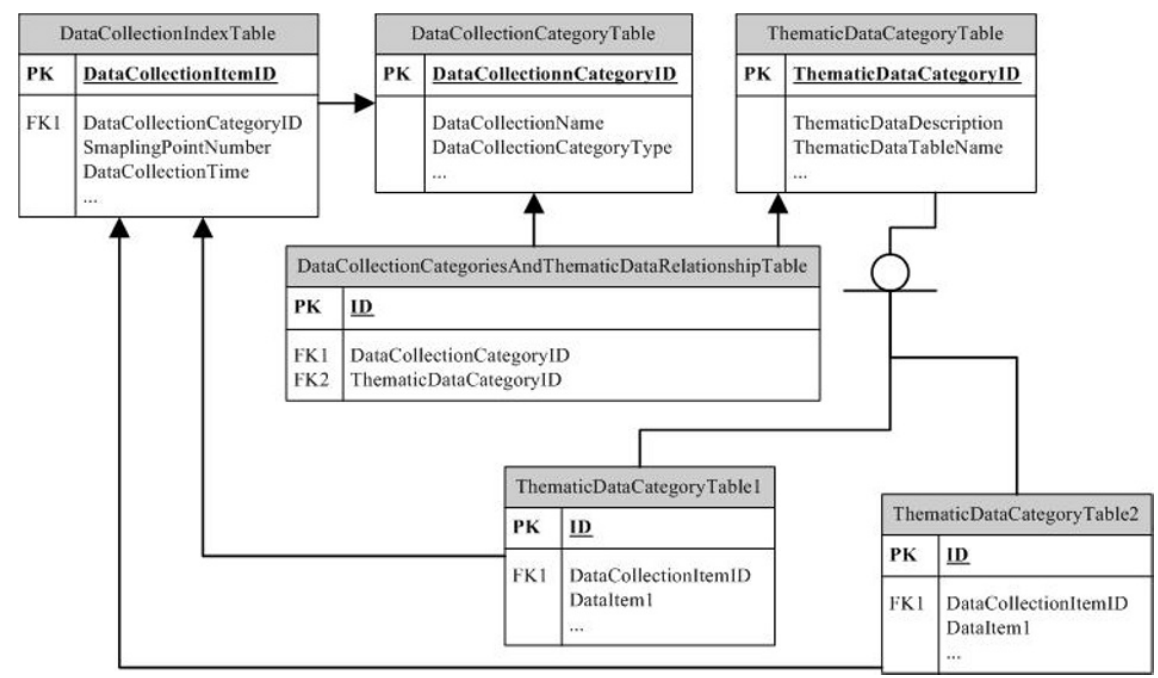

Figure 3. the relationship model of the main data tables

\subsection{Workflow and messaging format}

\subsubsection{Workflow}

Passive mode's data centers and data collection node's working process described as follows:

(1) launch and initialization;

(2) in the $4000 \mathrm{TCP}$ port monitoring connection requests of data collection node;

(3) receive connecting requests of data collection nodes, establish TCP connection;

(4) wait for data collection nodes' next round request of a data collection;

(5) receive data collection nodes' next round request of a data collection, send a welcome message;

(6) search database, send data acquisition options to data collection node; wait for feedback;

(7) receive information containing data from data collection node, analyze and order;

(8) continue to perform from (6) until the completion of an acquisition;

(9) save the data of the current round of collection into the database, go to (4);

(10) in this process, if received request of closure from data collection nodes, close the connection, go to (2). 


\subsubsection{Message format}

(1) there are three types of information from data central node to data collection node: welcome information, information of data option and finished information. Welcome information is the first information given to clients at the beginning of the acquisition, which contains tips for users' option and data option is options of the available data; finished information is the instruction of the end of an acquisition. Message format in Figure 4:

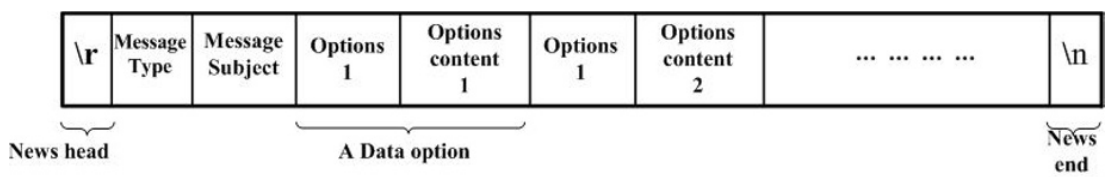

Figure 4. Data central node message format

' $\backslash \mathrm{r}$ ' and ' $\backslash \mathrm{n}$ '. Respectively, stands for the head and the end.

Information types, 0: welcome information; 1: data options; 9: Acquisition completed.

Information theme, a summary of the content of the message. Type 1, the theme of the content of data options.

Options identifier, only for type 1 , is the identifier for users to choose an option of data.

Options content, when type 1 , this is for users to choose an option of a data content.

For example, data collection nodes in the choice of "data collection categories," the feedback information is "soil nutrient acquisition," then the data center node retrieves "data acquisition categories and thematic data relationship Table" and " thematic data categories table ",there is an information in Figure 5. In this case options identifier is "thematic data Category ID" in "thematic data categories table".

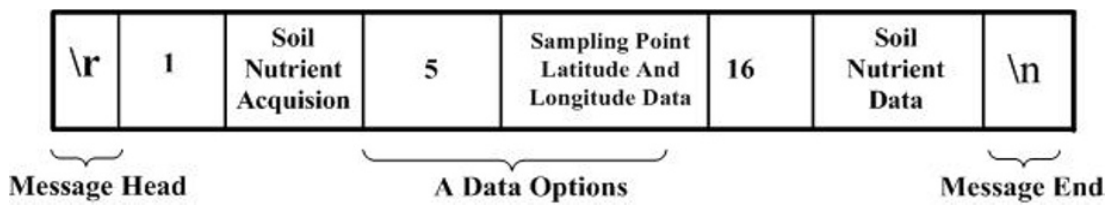

Figure 5. Data central node message format example

(2) The client gives server two kinds of information: requests to begin information collection and data contained information. Message format see Figure 6. When type is 0 , that is requesting begin information; when it is non- 0 , there is the data information which is the option identifier for users to 
select. Only when "information type" is non-0, the data type is effective. If its value is 00 , then the information is the collected data.

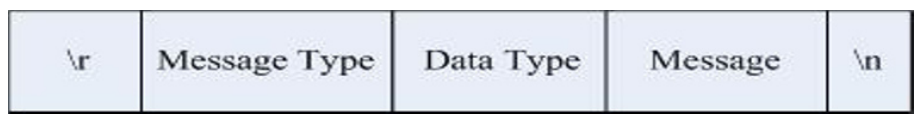

Figure 6. Data collection node message format

\subsection{Business interface layer}

The data center node provide business systems the following functions: (1) to provide data collection log list based on "data acquisition catalog" (2) to provide associated data according to the business system's choice of the data items of "data acquisition catalog table". Release these functions to Web services to form the business interface layer of data center node.

\subsubsection{Data service components}

The main components of data services include: (1) DataServiceDAO, to abstract bottom data to access. (2) DataServiceXML, to provide data object XML mapping for DAO, and to construct XML documents to a string format. (3) DataServiceValueObject, to provide the value objects of table of entities. (4) DataServiceSession, is no-condition conversation Bean, which packages mutual operations with other data service components, acts as the agent of business systems. Remote interface DataServiceSession defines the interface of Web services, interface definition is as follows.

/ **

* this is the remote interface for the System Session EJB

$* /$

public interface DataServiceSession extend EJBObject \{

public String getDataCollectionList () throws RemoteException;

public String getSpecialData (int dataCollectionItemID) throws RemoteException; \}

\subsubsection{Web services}

Use BEA WebLogic servicegen to make DataServiceSession EJB form interface of services based on JAX-RPC (Naveen, et al. 2007; Mikhail,2007) and deployment documents, and package it as the deployable EAR files. Take business system's data acquisition directory as an example, data services components' interactive sequence show in Figure 7. 


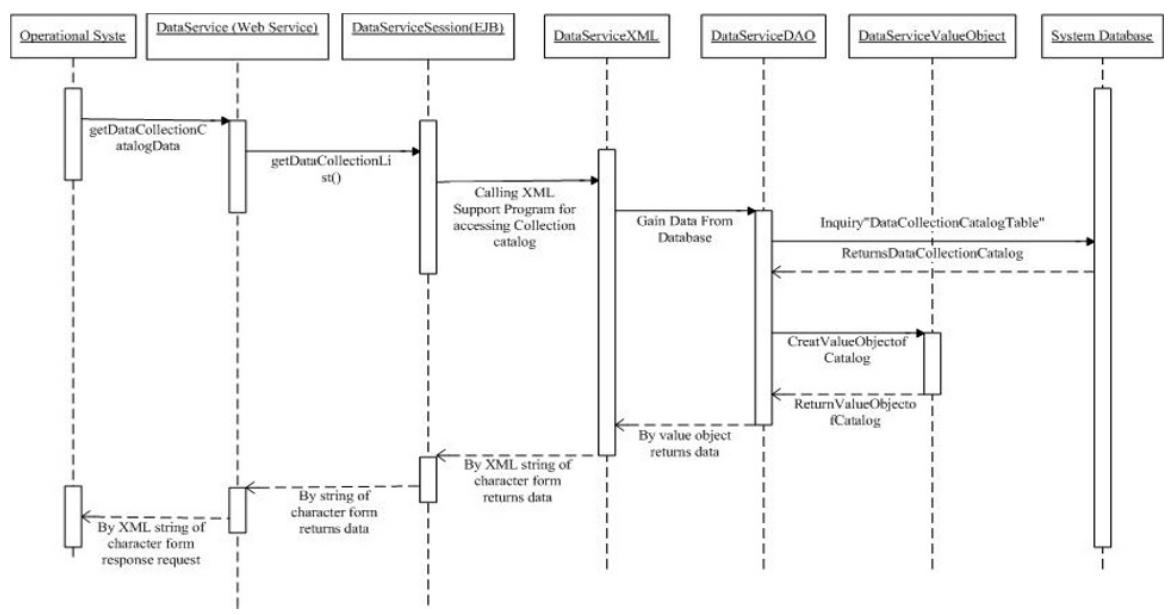

Figure 7. Data services components' interactive sequence

\section{DATA COLLECTION NODES DESIGN}

Node adopts embedded system and uses Borland $\mathrm{C}++3.1$ integrated development environment. Hardware includes: NetBox-II development board, $4 \times 5$ digital keypad and $320 \times 240$ LCD screen. Through the board integrated serial interface, it can realize the multi-source data collection to increase ETA197 data acquisition module (ETA197,2006). To increase Siemens MC39i GPRS communications module or WAVECOM Q2358C CDMA communications module and the board integrated Ethernet interface can achieve the support of variety of links. In addition NetBox-II provides a TCP / IP and PPP-TCP/IP protocol stack, which can be convenient link to variety of network-based application development.

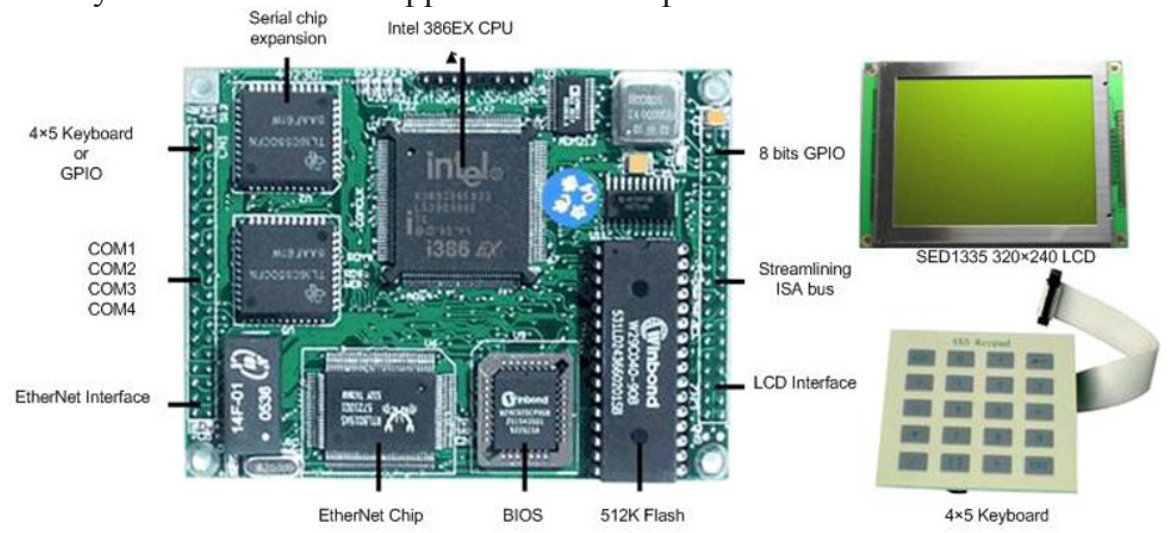

Figure 8. NetBox-II development board, LCD and Keyboard 


\subsection{Module structure}

The main function modules include : (1) keyboard interface module, responsible for receiving the user's keyboard input, making filtering process, sending it into the keyboard buffer zone, and returning to the LCD screen. (2) the interface showing module, due to the NetBox-II of the screen's showing problem, users can not directly use development tools' base functions to screen out Chinese characters, they need to use the specific driver function of specific LCD to dealing with characters' display, in which character dealing Module is used to initialize LCD screen, load English and Chinese characters to allow the system to support the Chinese characters. (3) Network processing module, responsible for processing network transmission-related work, including start-up / shut down Ethernet / GPRS / CDMA interface, create / release of TCP connection, receiving / sending TCP packet, and so on. (4) Information processing module, responsible for processing information interacted with data center node. Data collection node module structure show in Figure 9.

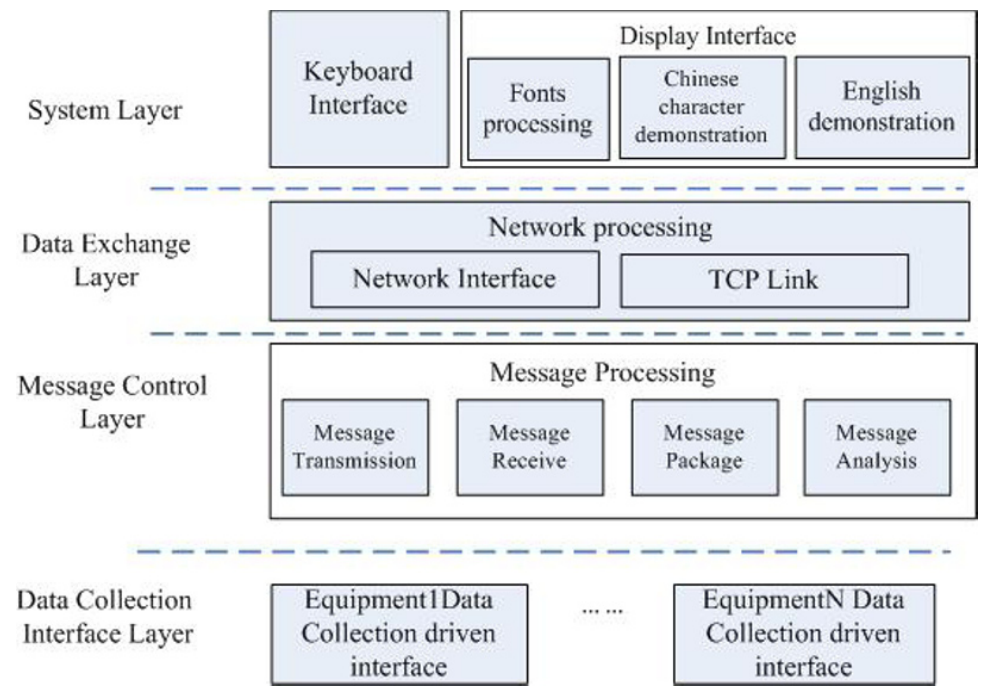

Figure 9. Data collection node module structure

\subsection{Workflow and status conversion}

The main cycle of data collection node complete receiving and processing data center node information, processing keyboard input and sending messages. The workflow show in Figure 10. In each cycle it deals with this three-part work orderly. To carry out mutually exclusive three-part work, a 
state variable ' $\mathrm{ns}$ ' is introduced to record the client running state, and to give different treatment in accordance with different states.

Data collection node running states are showed as the main and sub-state, the main state has four kinds of circumstances: EP_IDLE, EP_SVR, EP_FDBK and EP_QUIT, the sub-state has two kinds of circumstances: ING and ED, the status conversion of data collection node see Figure 11.

EP_IDLE: idle. When the state is ING, wait for users to start a keyboard order of collection or a keyboard order to withdraw from the system, deals only with the keyboard input, not to receive information. If received user's acquisition orders at this time, construction begins requesting information and sent into the buffer zone, the sub-status changes into the ED, after sending messages has been completed, the main state changes into EP_SVR; If user presses the exit button, the main state changes to preparing for Exit status EP_QUIT.

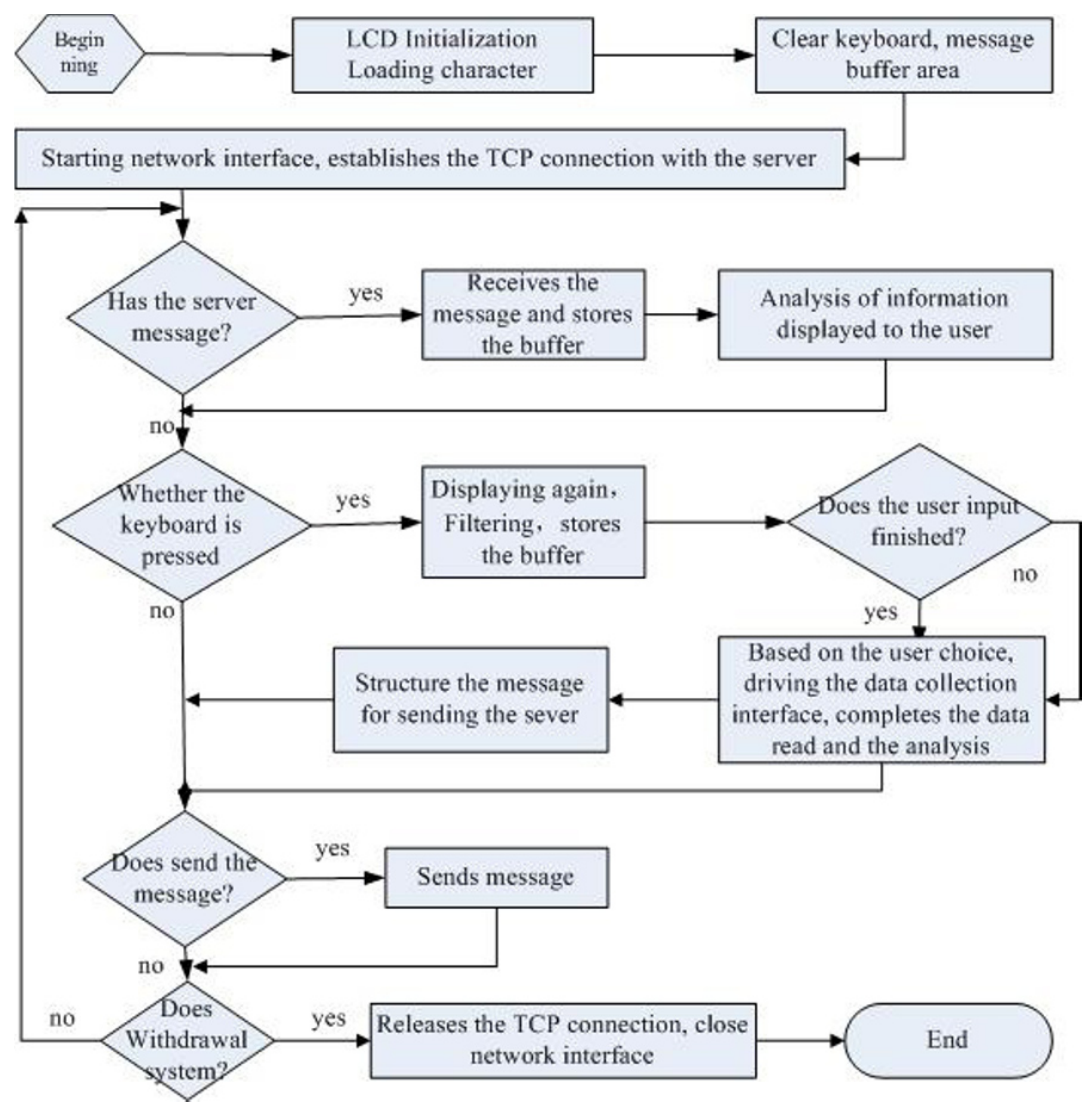

Figure 10. workflow chart 
EP_SVR: waiting for receiving information of data center node status. At this time, a collection has already begun; it can receive information from the data central node or the suspension keyboard orders of user. If it receives data option information of server data center node, analyzes and outputs to the LCD screen, the main state changes into EP_FDBK, waiting for users to input data from the keyboard; if the user presses withdraw, the main state will change into preparing for exit status EP_QUIT.

EP_FDBK: waiting for users to input data from the keyboard. If the substate is ING, to receive the user's keyboard input and sent into the keyboard buffer zone, when the input has been completed, the status will change into the ED. At this time, construct the data sending to the central node of data sources, sent them into the buffer zone, wait for sending.

EP_QUIT: Preparing for withdraw. If the main status is EP_IDLE, and the sub-status is ING, the user can press the ESC key to lead this system into this state, wait for the after-treatment and withdraw from the system.

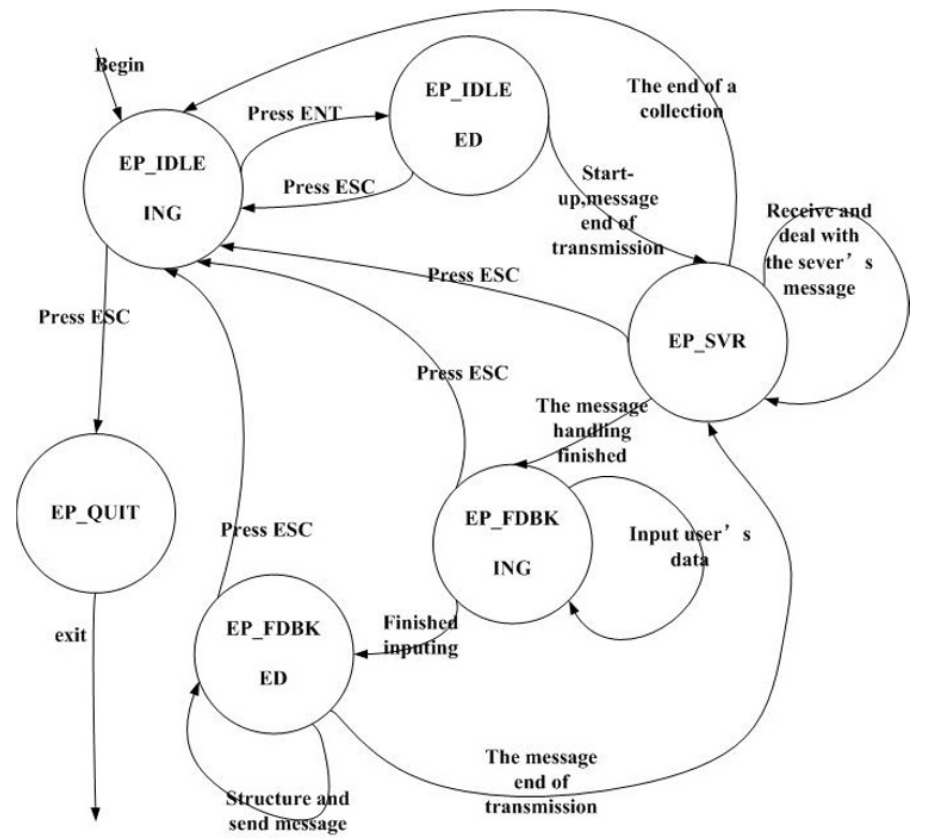

Figure 11. The status conversion of data collection node

\subsection{Serial signal acquisition}

To send and receive serial communication data of RS232/RS485, in the course of system development to see the serial signal providers as serial signal acquisition targets, to abstract a serial port on each object to a serial 
signal acquisition drive category. Such a package: (1) int InitUART (int ComIdx, int BaudIdx, int Parity), completed serial initialization; (2) int InstallISR (int ComIdx), serial port interrupt settings, (3) int PutOutputData (int ComIdx, char abyte), the data will be sent into output data buffer zone. (4) void StartSend (int ComIdx), launching interrupted, the sending data process begins (5) int GetInputData (int ComIdx), to retrieve data from the receiving data buffer zone, analyze the collection of data, form a format data. In addition, could add ETA518 expansion boards (ETA518,2006) to achieve timer interrupt serial operation.

\section{CONCLUSION}

The embedded agricultural origin data acquisition system developed in this research has the following characteristics (1) The realization of multisource data collection, data transmission based on a variety of physical link and the opening of the data service interface integration, can provide the technical support for monitoring pollution-free agricultural products or green Agricultural production (2) it has a good scalability and adaptability. With proper adjustments to hardware and software of the system, it can be easily applied to other areas of industry data' collection work. In practical application, the system provides the monitoring data of agricultural production environment for pollution-free agricultural products digital authentication system, and realizes the combination of the survey and directions for soil expert system (Xi, et al.2005), which could provide the sampling point's space data and soil nutrient data.

\section{ACKNOWLEDGEMENTS}

This study has been funded by China National 863 Plans Projects (Contract Number: 2006AA10Z270-4) and. It is also supported by the Education department of Henan province Scientific and technological projects (Contract Number: 200510466005).

\section{REFERENCES}

ETA197. http://www.emtronix.com.cn/download/ETA197.pdf ETA518. http://www.emtronix.com.cn/download/ETA518.pdf

Fan Li, Ding Zhuyu, Yu Guangwei, Huang Jie, Zhou Yinghua. Research of the Application of Embedded GPRS Long-Range Transmission Technology in Accurate, Agriculture Journal 
of Southwest University(Natural Science Edition), 2007,05 Vol.29,NO 5.pp137-140 (in Chinese)

Joann Wilcox. Embedded systems. Successful Farming. (Iowa edition). Des Moines: Apr 1998. Vol. 96, Iss. 6; pg. 19, 1 pgs

Kevin B. Mayer, Dr Ken Taylor CPEng, MIEAust. An Embedded Device Utilizing GPRS for Communications. ICITA2002.

Meng Hong.Trend of the technology of agricultural information collection, Agriculture Network Information,2006,12,NO 12.pp35-38 (in Chinese)

Meng Zhijun, Wang Xiu, Zhao Chunjiang, Xue Xuzhang. Development of field information collection system based on embedded COM-GIS and pocketPC for precision agriculture , Transactions of The Chinese Society of Agricultural Engineering 2005,04 Vol.21,NO 4.pp 91-96 (in Chinese)

Meng Zhijun, Zhao Chunjiang, Wang Xiu, Chen Liping, Xue Xuzhang. Field multi-source information collection system based on GPS for precision agriculture, Transactions of The Chinese Society of Agricultural Engineering,2003,07 Vol.19,NO 4. pp13-18 (in Chinese)

Naveen Balani, Rajeev Hathi. Design and develop JAX-WS 2.0 Web services. https://www6.software.ibm.com/developerworks/education/ws-jax/index.html, 2007,09

NetBoX-II. http://www.emtronix.com.cn/download/NetBox-II_low_price.pdf

Qiu Huanguang, Deng Xiangzheng, Zhan Jinyan, Guo Zaocheng. Design of a distributed data acquisition and spatial decision making system for precision agriculture. Transactions of The Chinese Society of Agricultural Engineering 2005,10 Vol.21,NO 10.pp109-113 (in Chinese)

Wang Qian, Sun Zhongfu, Li Xiuhong, Zhang Hongtao, Wang Yingchun. Agricultural Environment Monitoring Based on Embedded System,Control \& Automation, 2006,Vol.23,NO 8-2.pp38-41 (in Chinese)

Xi Lei, Ma Xinming, Hu Xiaohong, Zhao Hao, Li Jing. Agriculture Intelligent Decision System Development Platform Based On Knowledge And Growth Model, Journal of Shanxi University of Science \& Technology, 2005,12 Vol.23,NO 6.pp113-117 (in Chinese)

Xiao Chunhua, Li Xiuhong, Sun Zhongfu, Zhang Hongtao, Song Zikai.Implementation of a wireless and remote environment monitoring system, Microcomputer Information, 2007,11 Vol.23,NO 4-2. pp 4-6 (in Chinese)

Zhou Guoxiang, Zhou Jun, Miao Yubin, Liu Chengliang. Development and application on GSM-based monitoring system for digital agriculture, Transactions of The Chinese Society of Agricultural Engineering, 2005, 06. Vol.21, NO 6. pp87-91 (in Chinese) 